\title{
Modulatory Effect of Acetaminophen-Oxicodon and Lorazepam on the Pain Elicited by Needling in Electromyography
}

\section{Hamed Taheri ${ }^{1,2 *}$, Hamed Amiri Fard², Elham Sarhadi ${ }^{2}$}

${ }^{1}$ Department of Internal Medicine, Ali-Ebn-Abitaleb Hospital, Zahedan University of Medical Sciences, Zahedan, Iran ${ }^{2}$ School of Medicine, Zahedan University of Medical Sciences, Zahedan, Iran

\section{Article Info:}

\section{A BSTRACT}

Introduction: Pain is one of the complications of electromyography. The present study was designed to evaluate the effects of acetaminophen-oxycodone and lorazepam on the pain caused by needling in electromyography. Materials and Methods: In this clinical study, 150 patients were divided into three groups (receiving lorazepam, acetaminophen and placebo). Pain was measured by visual analogue scale. Results: There was no significant difference between acetaminophen and lorazepam groups. Conclusion: there is no difference between lorazepam and acetaminophen on the pain induced by electromyography.

*Corresponding Author: Hamed Taheri

E-mail: Dr.taheri.113114@gmail.com 


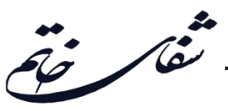

$$
\text { اثر تنظيمى استامينوفن اكسى كدون و لورازيام بر درد ناشى از الكترومايوكرافى سوزنى }
$$

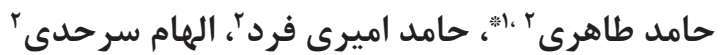

$$
\begin{aligned}
& \text { اكروه داخلى، بيمارستان على ابن ابيطالب (ع)، دانشكاه علوم يزشكى زاهدان، زاهدان، ايران } \\
& \text { ז'دانشكده يزشكى، دانشخاه علوم يزشكى زاهدان، زاهدان، ايران }
\end{aligned}
$$

\section{اطلاعات مقاله:}

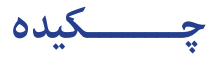

مقدمه: درد يكى از عوارض ناشى از الكتروميوگرافى مىباشد. مطالعة حاضر بلمنظور ارزيابى تأثيرات

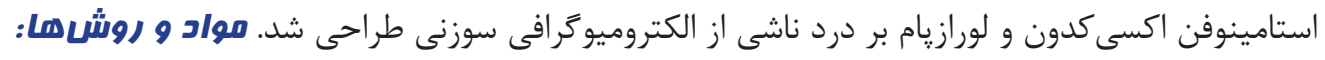

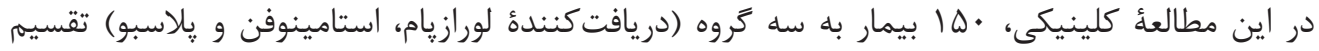

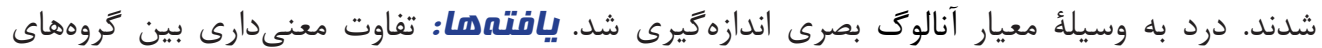

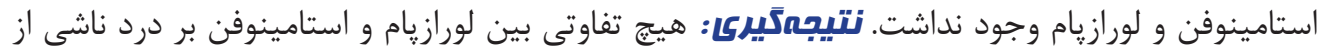
الكترومايوگر افى وجود ندارد.

كليد وازمها:

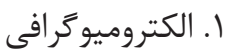

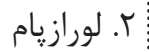

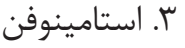

د أl

" نويسنده مسئول: حامد طاهرى آدرس الكترونيكى: Dr.taheri.113114@gmail.com 


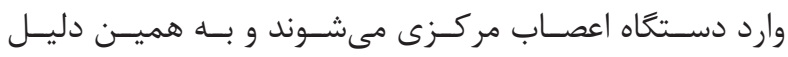

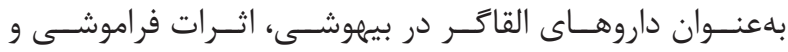

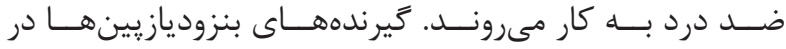

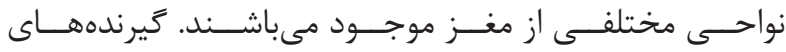

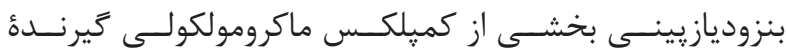

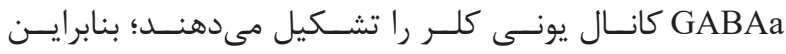

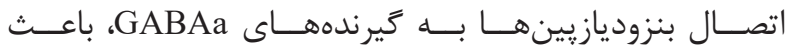

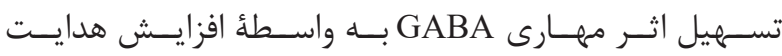

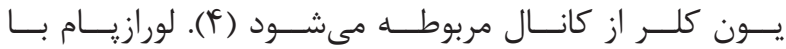

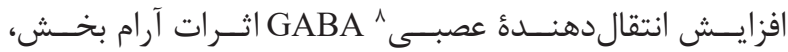

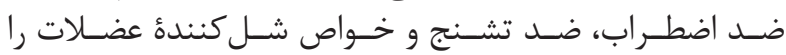

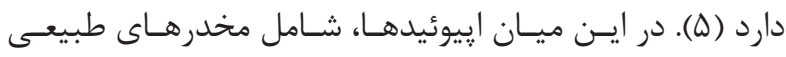

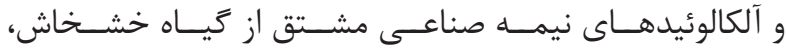

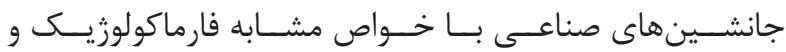

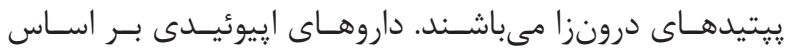

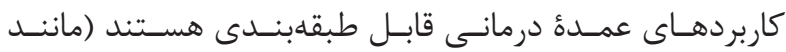

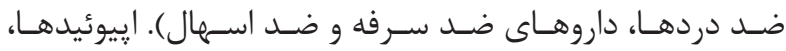

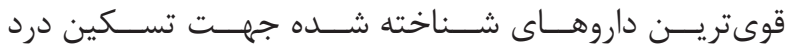

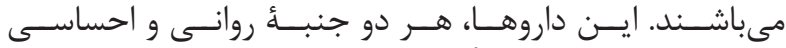

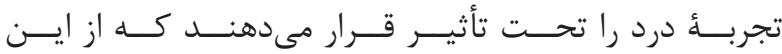

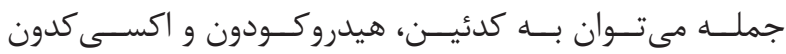

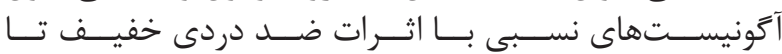

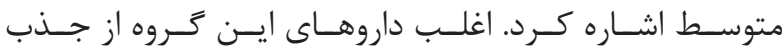

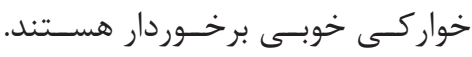

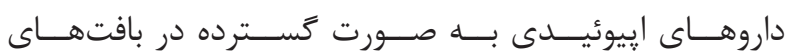

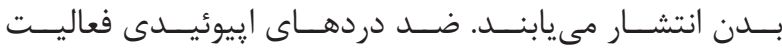

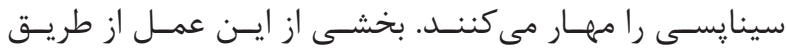

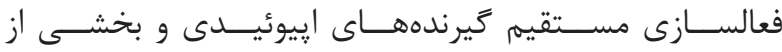

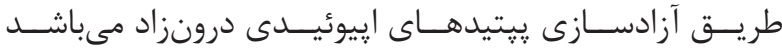

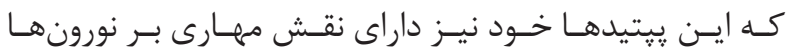

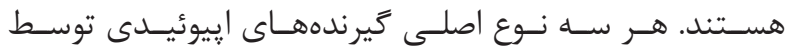

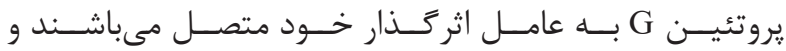

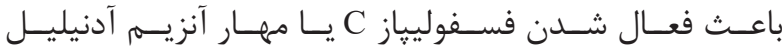

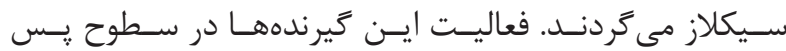

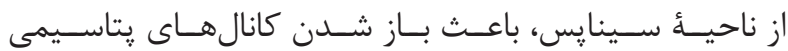

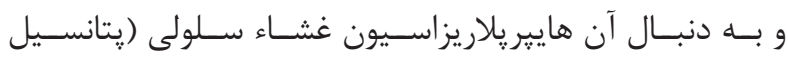

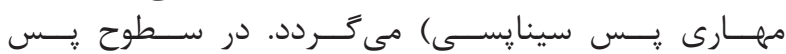

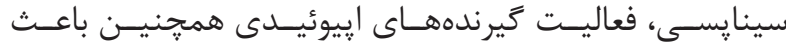

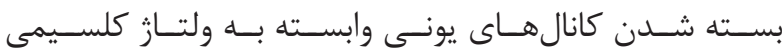

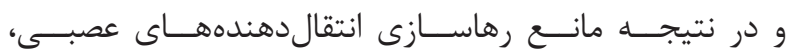

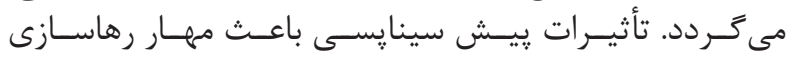

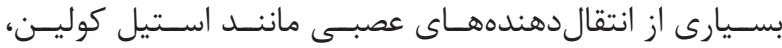

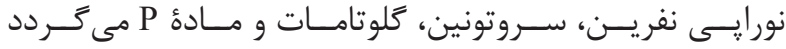

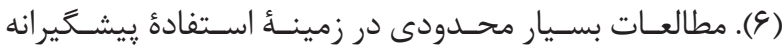

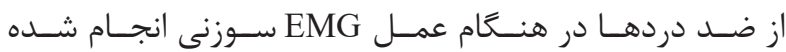

\section{${ }^{1}$ Duchenne}

${ }^{2}$ Beker

${ }^{3}$ Congenital myasthenic syndromes

${ }^{4}$ Charcot marie tooth

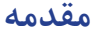

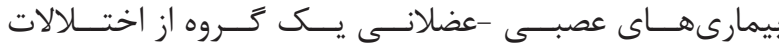

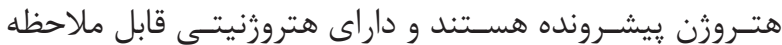

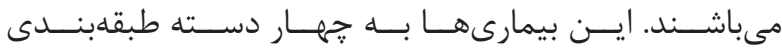

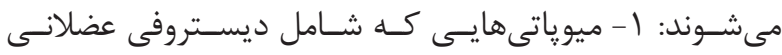

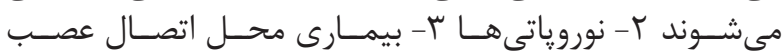

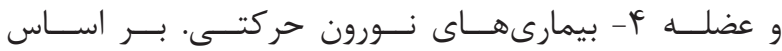

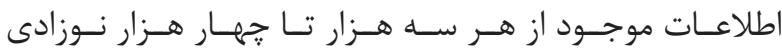

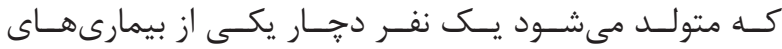

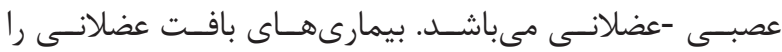

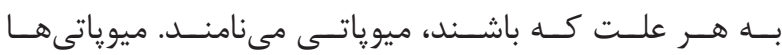

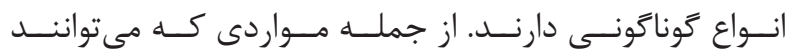

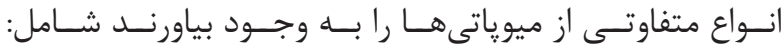

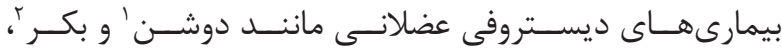

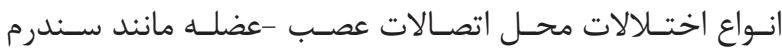

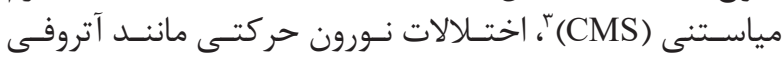

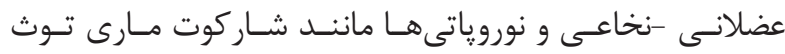

(1) (CMT)

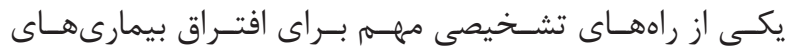

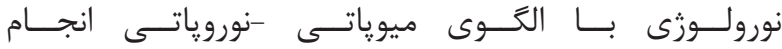

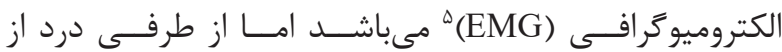

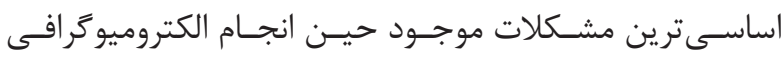

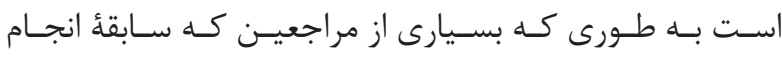

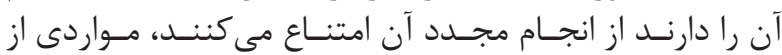

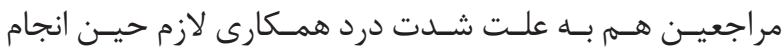

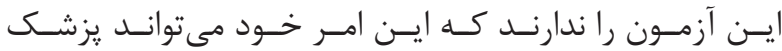

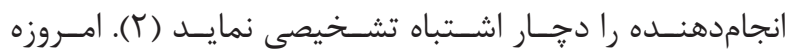

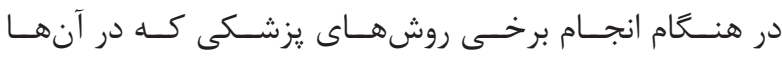

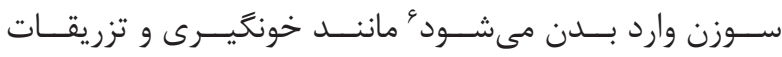

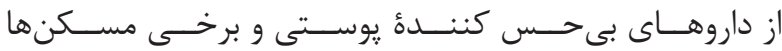

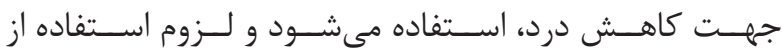

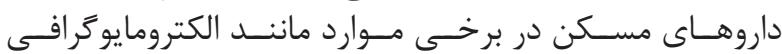

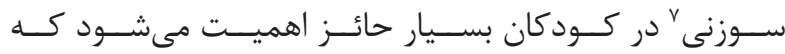

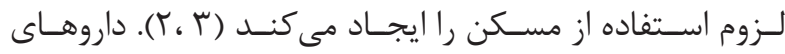

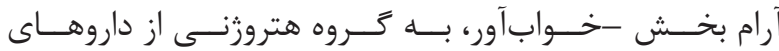

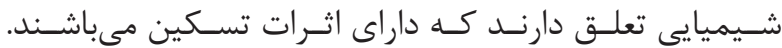

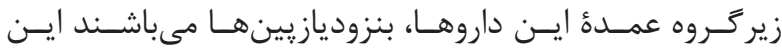

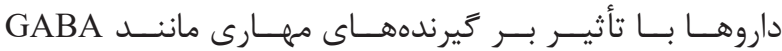

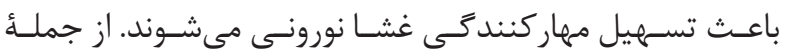

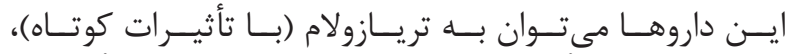

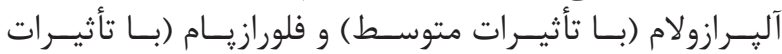

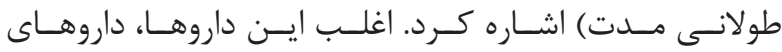

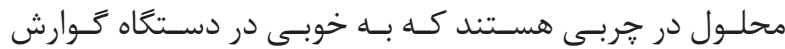

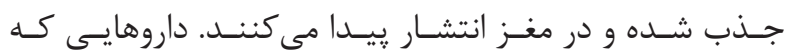

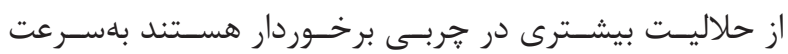

${ }^{5}$ Electromyographic

${ }^{6}$ Needling

${ }^{7}$ EMG needeling

${ }^{8}$ Neurotransmitter 


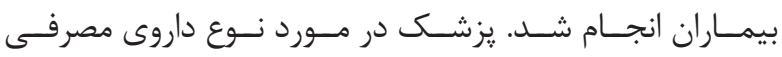

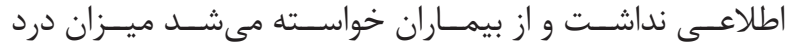

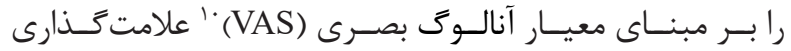

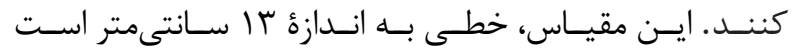

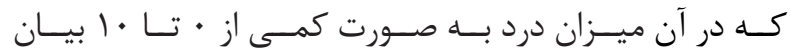

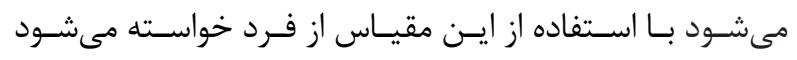

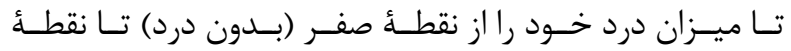

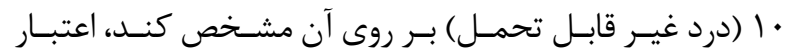

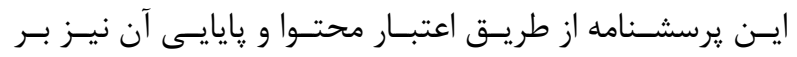

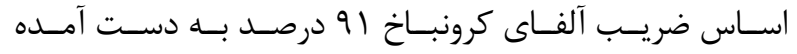

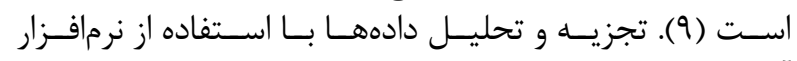

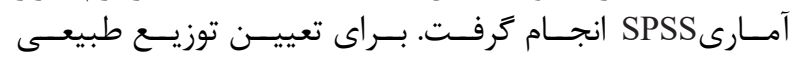

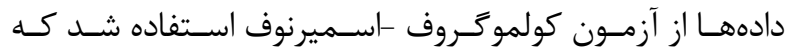

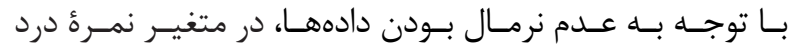

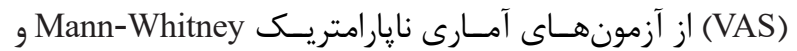
Kruskal-Wallis

بافتهها

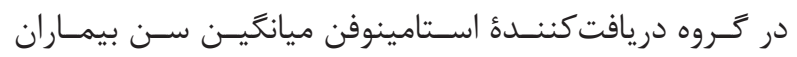
صه

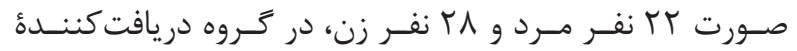

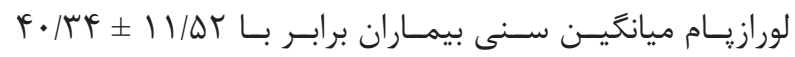

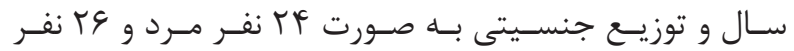

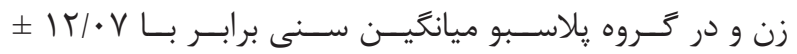

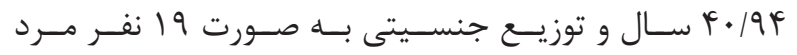

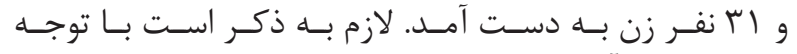

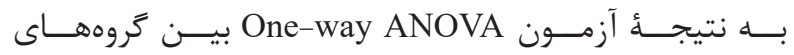

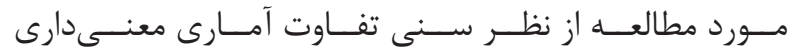

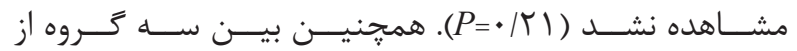

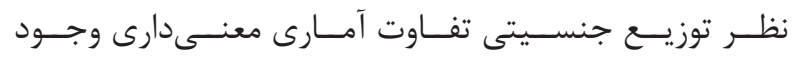

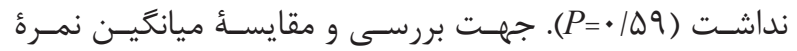

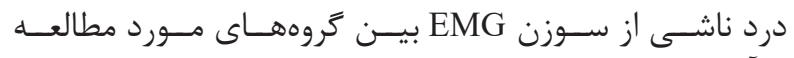

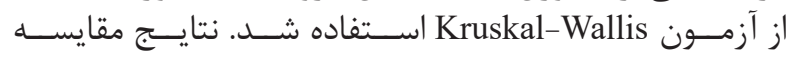

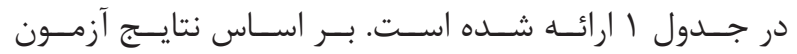

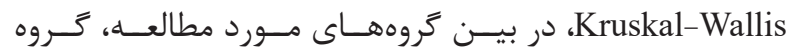

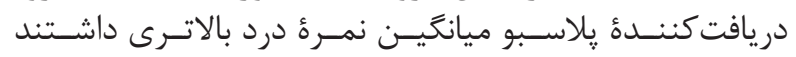

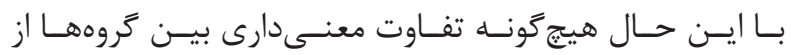

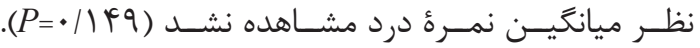

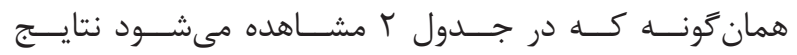

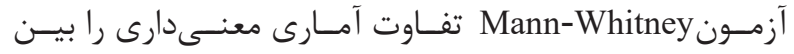

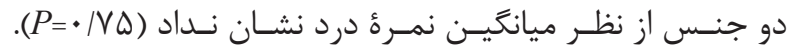

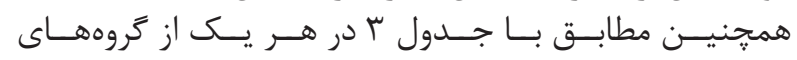

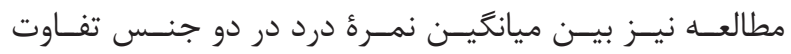

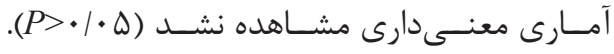

\section{بحث و نتيجه كيرى}

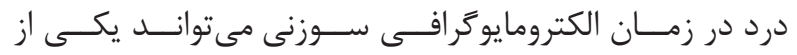

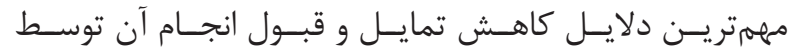

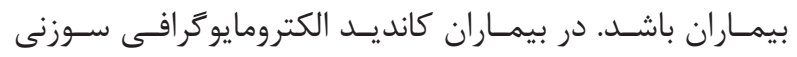

${ }^{9}$ Neurologist

${ }^{10}$ Visual analog scales

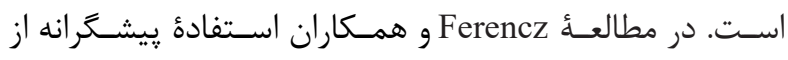

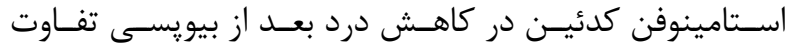

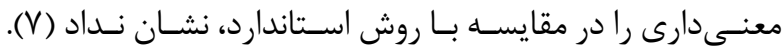

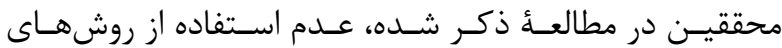

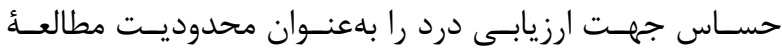

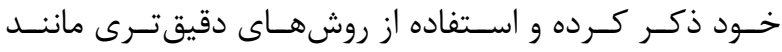

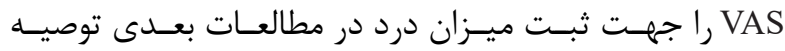

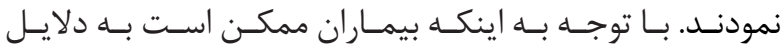

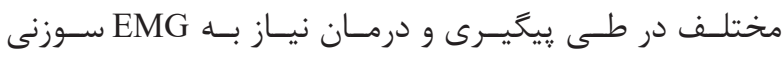

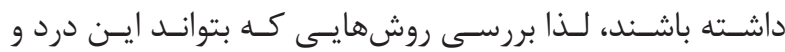

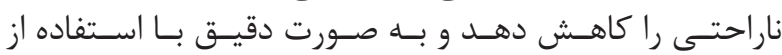

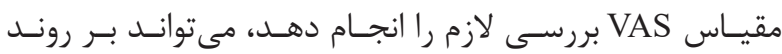

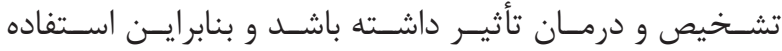

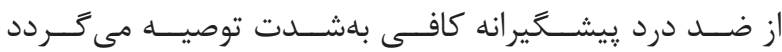

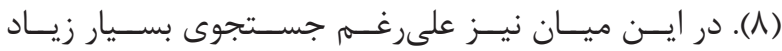

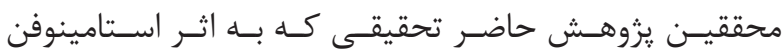

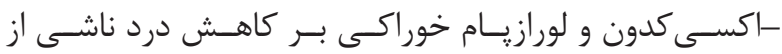

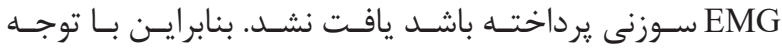

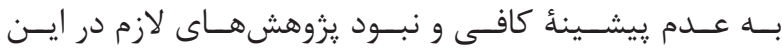

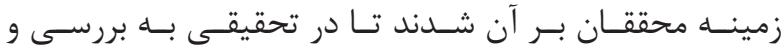

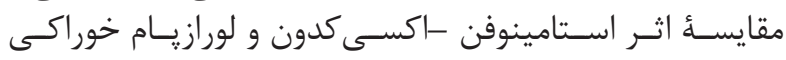

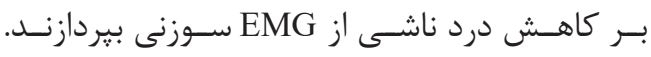

مواد و روشها

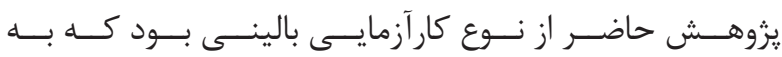

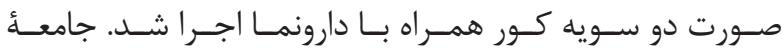

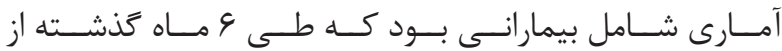

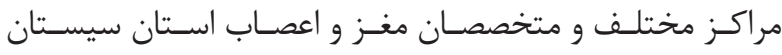

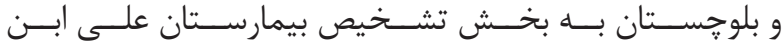

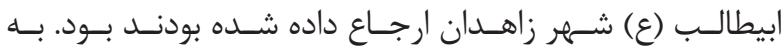

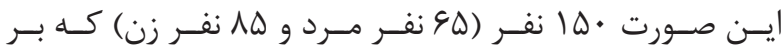

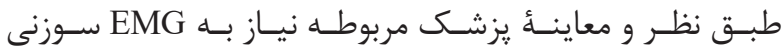

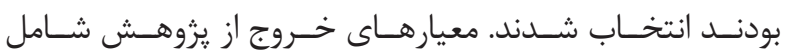

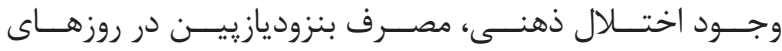

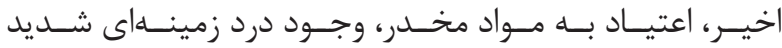

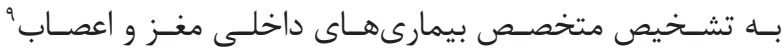

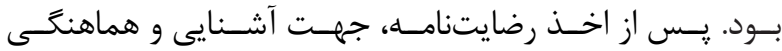

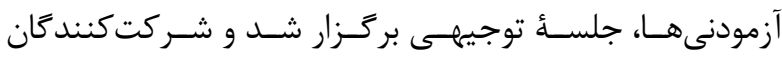

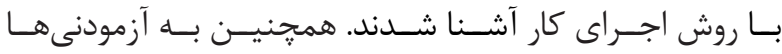

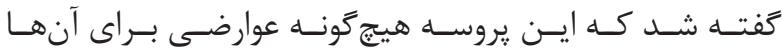

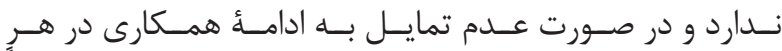

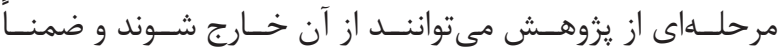

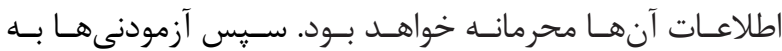

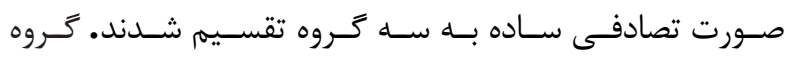

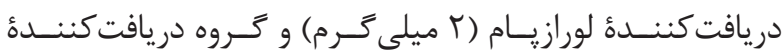

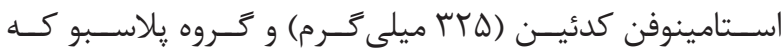

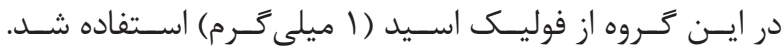

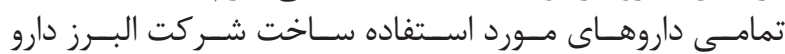

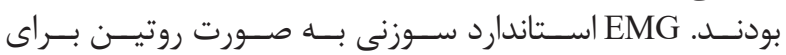


جدول ا- تعيين و مقايسٔ ميانكَين نمرة درد ناشى از سوزن EMG بين كروهها.

\begin{tabular}{|c|c|c|}
\hline سطح معنى دارى & نمرهٔ درد (Mean E SD) & تروه \\
\hline \multirow{3}{*}{$\cdot / 14 q$} & $\uparrow / \xi \wedge \pm 1 / V \Delta$ & دريافتكنندة استامينوفن \\
\hline & $f / F \varepsilon \pm 1 / \Lambda f$ & دريافتكنندة لورازيام \\
\hline & $\Delta / r \varphi \pm T / / 9$ & دريافت كنندة يّلاسبو \\
\hline
\end{tabular}

\begin{tabular}{|c|c|c|}
\hline سطح معنى دارى & نمرة درد (Mean I SD) & جنس \\
\hline \multirow{2}{*}{$\cdot / V \Delta$} & $r / R+r / \cdot r$ & مرد \\
\hline & $f / \Lambda f \pm 1 / 91$ & زن \\
\hline
\end{tabular}

جدول ب- تعيين و مقايسٔ ميانكَين نمرة درد ناشى از سوزن EMG بين دو جنس بر حسب كروه.

\begin{tabular}{|c|c|c|c|}
\hline سطح معنى دارى & نمرة درد (Mean \pm SD) & جنس & كروه \\
\hline \multirow{2}{*}{$\cdot / \mathrm{VA}$} & $f / V V \pm 1 / \Delta V$ & 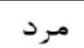 & \multirow{2}{*}{ دريافت كنندة استامينوفن } \\
\hline & $f / 9 \pm 1 / 91$ & زن & \\
\hline \multirow{2}{*}{$\cdot / \vee 9$} & $F / F \Delta \pm 1 / V \varepsilon$ & 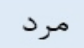 & \multirow{2}{*}{ دريافت كنـندة لورازٍام } \\
\hline & $f / f G \pm 1 / 9 f$ & زن & \\
\hline \multirow{2}{*}{.194} & $\Delta / \cdot \Delta \pm r / V r$ & 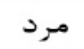 & \multirow{2}{*}{ دريافت كنندة يلاسبو } \\
\hline & $\Delta / \Gamma \wedge \pm \mid / \wedge)$ & زن & \\
\hline
\end{tabular}

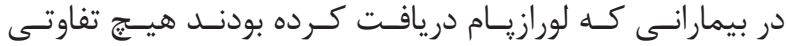

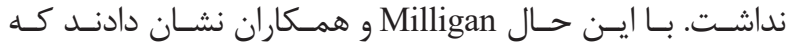

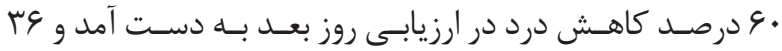

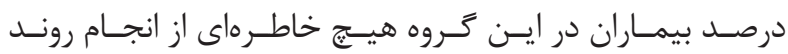

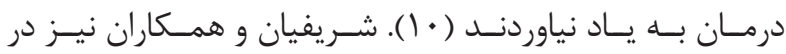

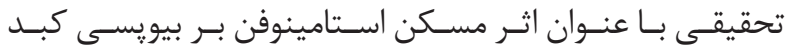

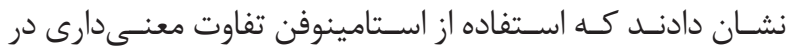

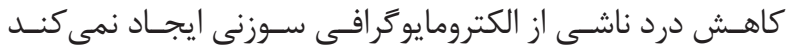

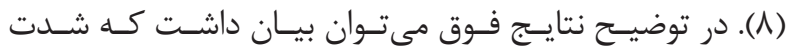

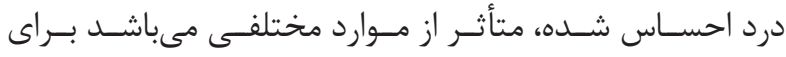

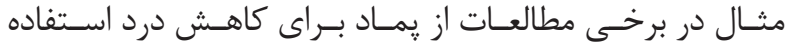

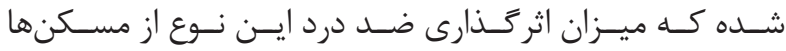

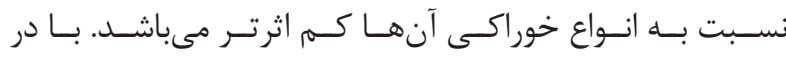

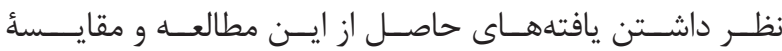

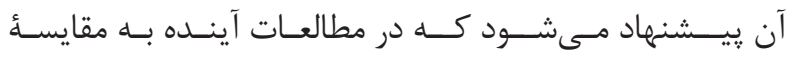

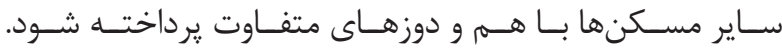

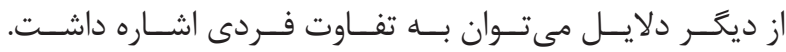

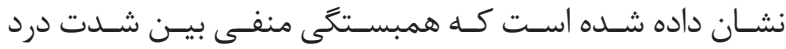

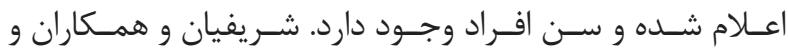

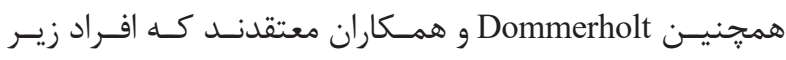

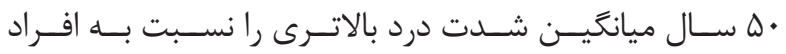

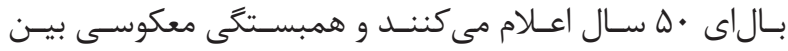

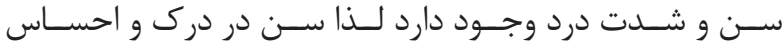

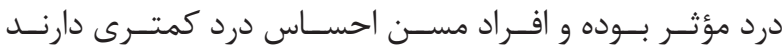

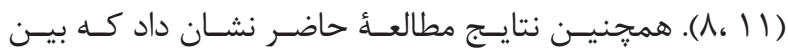

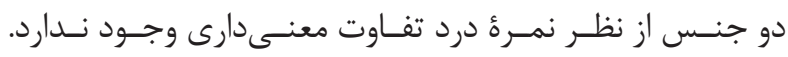

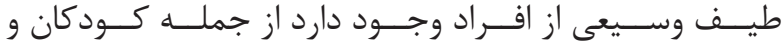

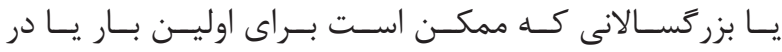

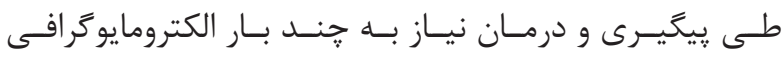

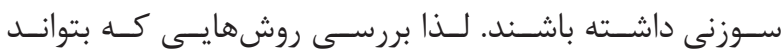

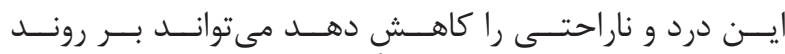

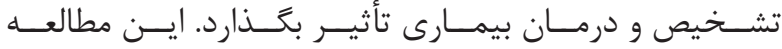

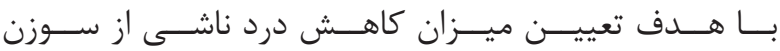

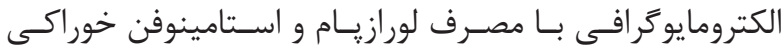

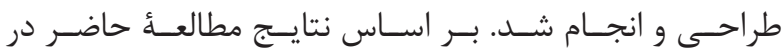

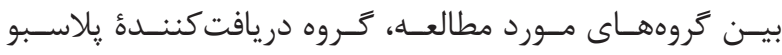

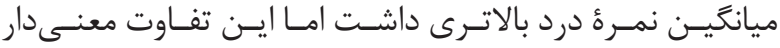

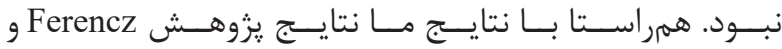

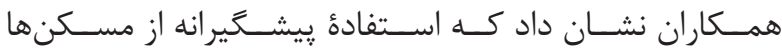

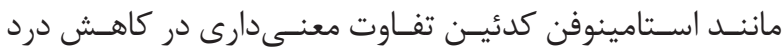

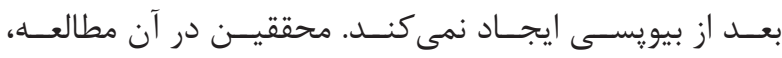

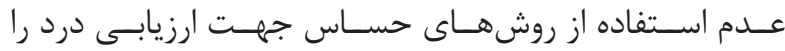

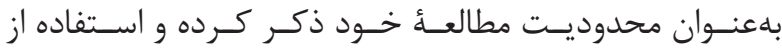

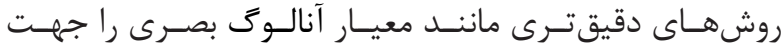

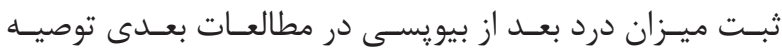

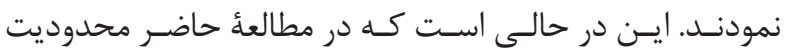

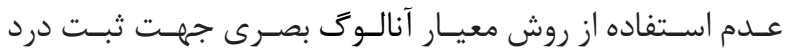

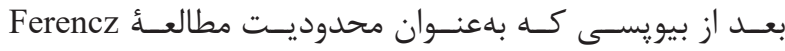

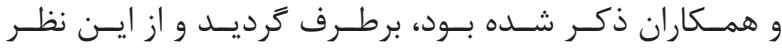

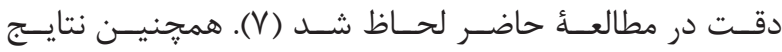

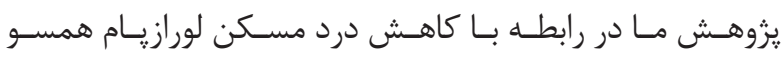

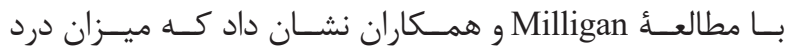


دادن توضيحـات و اطلاعـات لازم و كافـى در مـورد روش انجـام

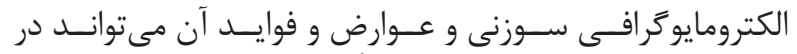

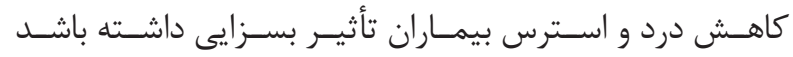

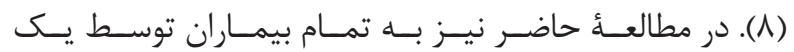

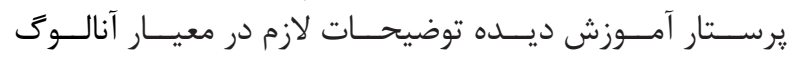

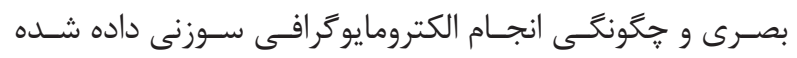

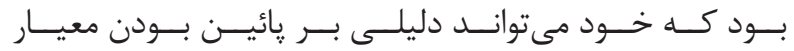

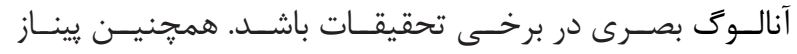

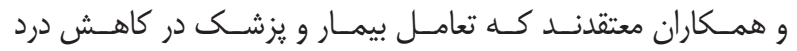

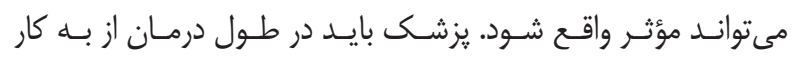

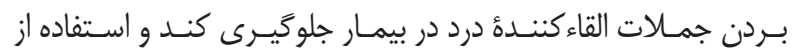

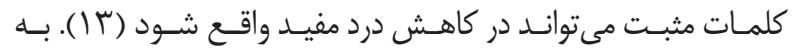

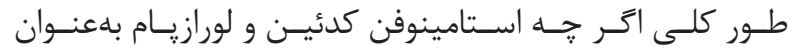

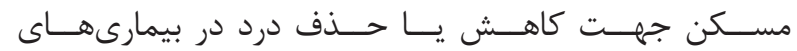

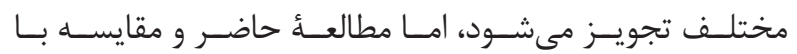

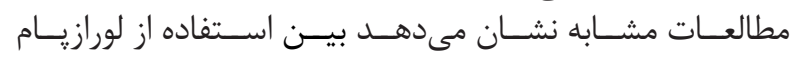

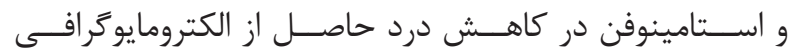

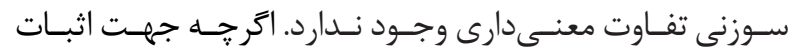

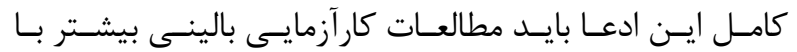

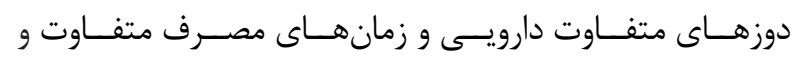

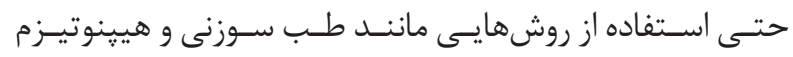
توصيــه مى حَــردد.

1. Kahrizi K, Shafaghati Y, Keyhani E, Hassan-Zad M, A'zimiyan M, Layeghi F, ea. Classification of Neuromuscular Disorders Based an Clinical Criteria , Molocular and Immunohistochemisty Analysis in Tehran Pateints. jrehab. 2005; 6(3): 44-8.

2. Bahrami MH, Rayegani SM, Azhari A, Moghimi HR, Valaei N, Dea E, et al. The efficacy of lidocaine$\mathrm{H}$ ointment in prevention of the pain associated with EMG- needling. Research in Medicine The journal of School of Medicine. 2010; 34(3): 152-6.

3. Dommerholt J, de las Penas CF. Trigger point dry needling: an evidence and clinical-based approach. J Can Chiropr Assoc. 2015; 59(1): 84.

4. Chiara DC, Jayakar SS, Zhou X, Zhang X, Savechenkov PY, Bruzik KS, et al. Specificity of intersubunit general anesthetic binding sites in the transmembrane domain of the human $\alpha 1 \beta 3 \gamma 2$ GABAA receptor. Journal of Biological Chemistry. 2013; 2: 19343-57.

5. Heydarifar S, Ilkanipour M, Farokhi F, M M. The effect of lorazepam and aqueous exetract of melissa officinalis on histological changes in the hippocampus and spitial memory in male rats. Qom University of Medical Sciences Journal. 2016; 10(4): 10-21.

6. Katzung BG, Masters SB, Trevor AJ. Basic and clinical pharmacology. 12 ${ }^{\text {th }}$ ed. (Lange Basic Science): McGraw-Hill Education; 2012.

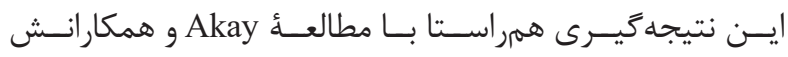

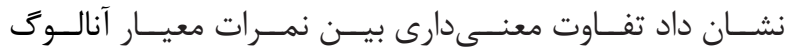

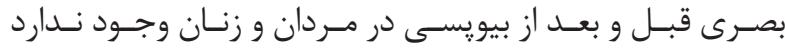

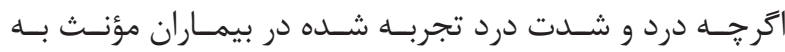

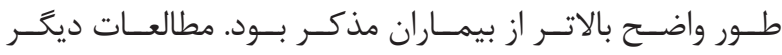

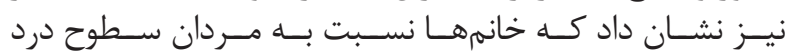

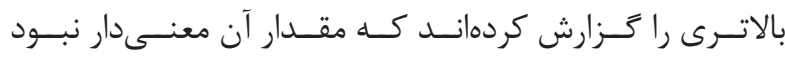

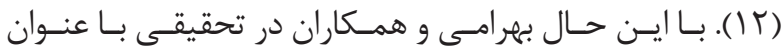

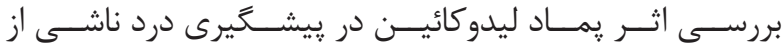

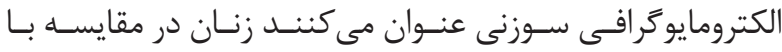

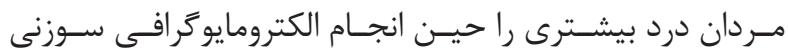

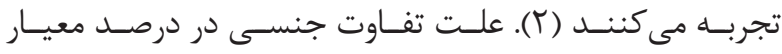

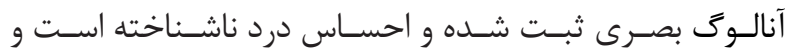

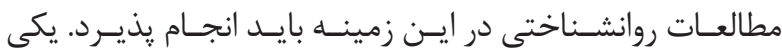

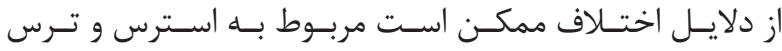

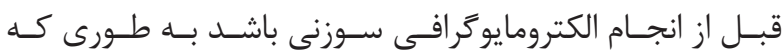

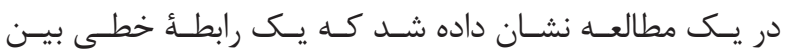

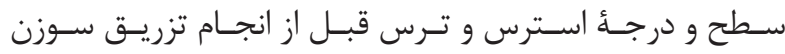

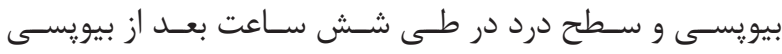

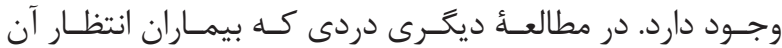

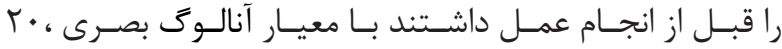

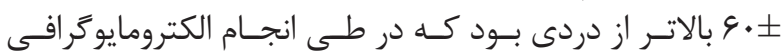

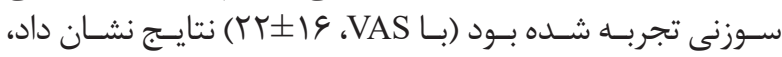
منابع

7. Ferencz S, Batey R. Can pre-emptive analgesia reduce pain experienced after liver biopsy? Intern Med J. 2002; 32(3): 122-3.

8. Sharifians F, Darwish N, Sabah H, Asadi B, Gharibi F, Allah Vezi A. The effect of prophylactic treatment of acetaminophen codein on pain after liver biopsy. Scientific Journal of Kurdistan University of Medical Sciences. 2009; 14(2): 37-44.

9. Sarhadi S, Ramezani A, Gholami M, Taheri H. The effect of an aerobic training cycle in the morning and evening on primary dysmenorrhea and some physiological variables in matured girls. Iranian Journal of Obstetrics, Gynecology and Infertility. 2015; 18(1): 20-11.

10. Milligan D, Howard M, Judd A. Premedication with lorazepam before bone marrow biopsy. J Clin Pathol. 1987; 40(6): 696-8.

11. Dommerholt J, de las Penas CF. Trigger point dry needling e-book: an evidence and clinical-based approach: Elsevier Health Sciences; 2018.

12. Akay S, Karasu Z, Noyan A, Pala S, Musoglu A, İlter T, et al. Liver biopsy: is the pain for real or is it only the fear of it? Dig Dis Sci. 2007; 52(2): 579-81.

13. Fernández-de-Las-Peñas $\mathrm{C}$, Nijs J. Trigger point dry needling for the treatment of myofascial pain syndrome: current perspectives within a pain neuroscience paradigm. J Pain Res. 2019; 12: 1899-911. 